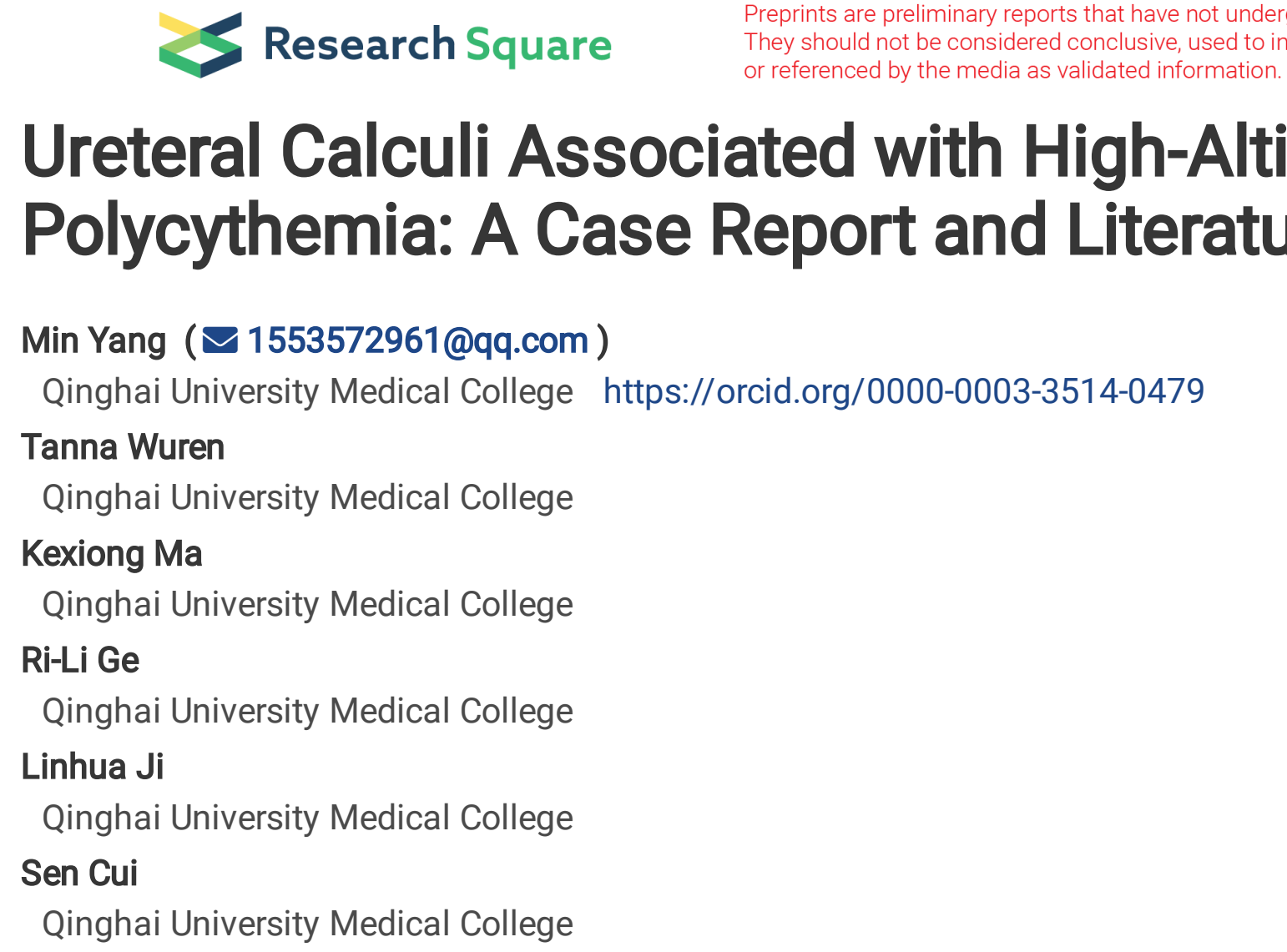

\section{Min Yang ( $\sim 1553572961 @ q q . c o m)$}

Qinghai University Medical College https://orcid.org/0000-0003-3514-0479

\section{Tanna Wuren}

Qinghai University Medical College

\section{Kexiong Ma}

Qinghai University Medical College

\section{Ri-Li Ge}

Qinghai University Medical College

\section{Linhua Ji}

Qinghai University Medical College

\section{Sen Cui}

Qinghai University Medical College

\title{
Ureteral Calculi Associated with High-Altitude Polycythemia: A Case Report and Literature Review
}

\section{Case Report}

Keywords: High-altitude polycythemia, ureteral calculus, hyperuricemia, case report

Posted Date: October 8th, 2020

DOl: https://doi.org/10.21203/rs.3.rs-87058/v1

License: (c) (i) This work is licensed under a Creative Commons Attribution 4.0 International License. Read Full License 


\section{Abstract}

Background: High-altitude polycythemia (HAPC) is a common disease in high-altitude areas characterized by excessive erythrocyte proliferation and severe hypoxemia. Recently, the incidence of ureteral calculi has a rising trend. However, cases of ureteral calculi associated with high-altitude polycythemia have not been reported.

Case Presentation: We present the cases of two patients with HAPC who were born in the lowlands and worked in high altitudes and were admitted to the hospital with acute severe pain in the ureter as the first symptom. Urological examinations confirmed the presence of a ureteral stone. Interestingly, the biochemical tests showed elevated serum uric acid levels and the calculous component analysis suggested anhydrous uric acid. Recently, extensive research has demonstrated a significant correlation between hyperuricemia and high-altitude polycythemia. Therefore, we speculated that the occurrence of ureteral calculi among immigrants to the plateau might be related to hyperuricemia associated with highaltitude polycythemia.

Conclusions: This case report and literature review highlights that the prevention of ureteral calculi in patients with polycythemia who immigrate to the plateaus from high-altitude areas should be considered. Additionally, the serum uric acid levels and urine $\mathrm{pH}$ should be monitored regularly.

\section{Background}

High-altitude polycythemia (HAPC) is a clinical syndrome caused by the inefficient acclimatization to high-altitude hypoxic environments. It is characterized by excessive red blood cell proliferation and severe hypoxemia (1). Recently, extensive research has demonstrated a significant correlation between hyperuricemia and HAPC, as hyperuricemia is a common complication in patients with HAPC (2-4). Uric acid is deposited in the kidneys in the form of sodium urate crystals when its concentration is supersaturated in the bloodstream. Furthermore, a persistent low urine $\mathrm{pH}$ also plays a key role in the pathogenesis of urolithiasis (5). Under high-altitude hypoxia, uric acid and other acidic metabolites increase in patients with HAPC, leading to the precipitation of uric acid crystals and subsequent deposition in the kidneys. Once the ureteral calculus is formed in such patients, the renal excretory function is aggravated, leading to the enormous accumulation of uric acid thereby continuing a vicious circle. As medical assistance in the plateau areas is often limited, when patients with ureteral calculi do not receive timely treatment a series of irreversible consequences may result. Therefore, the prevention of ureteral stones should be considered in patients with polycythemia after migrating to the plateaus. However, no studies on ureteral calculi concurrent with HAPC have been reported. Therefore, we sought to present two cases of ureteral calculi in patients born in lowlands and who worked at high altitudes. We aimed to expand the literature on this disease and help clinicians to consider methods in preventing its development.

\section{Case Presentation}




\section{Case 1}

The first case was of a 26-year-old Chinese woman who was admitted to a hospital located at 4,300 m (Maduo county, China) with complaints of severe pain in the right lower abdomen. The pain was accompanied by nausea, vomiting, chest tightness, and shortness of breath. The patient was a PhD student who was born in a lowland area (1,100 m; Tianshui, China) and had moved to Maduo county 3 months earlier to conduct her scientific research. At that high altitude, she gradually developed shortness of breath, nocturnal sleep disturbance, and lip cyanosis. A clinical assessment revealed that she had developed tachycardia (pulse rate, $132 / \mathrm{min}$ ), decreased $\mathrm{SpO}_{2}(73 \%)$, and hypotension (blood pressure, $90 / 58 \mathrm{~mm}$ of $\mathrm{Hg}$ ). Due to the limited medical assistance in the area, she was immediately transferred to our hospital for further evaluation and treatment. A physical examination of the upper abdomen showed tenderness and percussion pain over the right renal region and the ureter. An abdominal horizontal position radiograph (KUB) showed a deformable area with a high-density shadow on the right upper segment of the ureter suggesting the presence of a calculi. A urological ultrasound showed that the spot separation of the right renal collecting system was $12 \mathrm{~mm}$. On the right upper segment of the ureter, approximately $20 \mathrm{~mm}$ away from the renal pelvis, a bright spot with a diameter of approximately $7 \mathrm{~mm}$ was detected along with an acoustic shadow. The aforementioned findings revealed right hydronephrosis and right upper ureteral calculi. A routine blood investigation showed an increased absolute erythrocyte count and hemoglobin level $\left(6.58 \times 10^{12} / \mathrm{L}\right.$ and $192 \mathrm{~g} / \mathrm{L}$, respectively). Further clinical biochemical tests revealed a high uric acid level $(458 \mu \mathrm{mol} / \mathrm{L})$. Routine urine examination was positive for occult blood $(2+)$ and ketone bodies (2+). The peripheral blood Janus kinase 2 (JAK2) gene test showed no significant abnormalities. After evaluation, the patient underwent extracorporeal shock wave lithotripsy. Analysis of stone components suggested anhydrous uric acid. Additionally, no recurrences were found upon regular re-examinations.

\section{Case 2}

The second case was of a 31-year-old Chinese man who was admitted to our hospital with complaints of right lumbar pain accompanied by gross hematuria for 3 days. The patient was born in a lowland area (47m; Yantai, China) and worked as an engineer in the highlands (2,994 m; Haixi, China) for 3 years. He had a 2-year history of HAPC, without receiving any treatment. A physical examination revealed a right renal and ureteral tenderness, slight cyanosis of the lips, telangiectasia of the face, and conjunctival hyperemia. A clinical assessment revealed that he had tachycardia (pulse rate, 118/min) and reduced $\mathrm{SPO}_{2}(79 \%)$. The patient's body temperature, respiratory rate, and blood pressure were within the normal limits. Ultrasonography of the urinary system indicated $11 \mathrm{~mm}$ separation of the right kidney collection system. The inner diameter of the upper part of the right ureter was $8 \mathrm{~mm}$, while the middle and lower part were not clearly displayed. The left ureter does not dilate. KUB showed no positive urinary calculi in the bilateral renal area and ureteral path. Intravenous urography (IVP) showed right hydronephrosis after intravenous contrast agent injection. There was dilatation in the upper and middle segment of the right 
ureter, and no dilatation, hydronephrosis or damage in the left ureter. Imaging diagnosis: the right middle and lower ureter incomplete obstruction was considered. A routine blood investigation revealed increased absolute erythrocyte count and hemoglobin levels $\left(7.78 \times 10^{12} / \mathrm{L}\right.$ and $225 \mathrm{~g} / \mathrm{L}$, respectively). Further clinical biochemical tests revealed high uric acid $(579 \mu \mathrm{mol} / \mathrm{L})$, and creatinine $(64 \mu \mathrm{mol} / \mathrm{L})$ levels. $A$ routine urine examination was positive for occult blood (3+) and protein (1+). The peripheral blood JAK2 gene test, tumor marker levels, and heart color doppler ultrasound results detected no significant abnormalities. The patient underwent right ureteroscopy and right ureteral stenting. After the operation, the patient was given anti-inflammatory, hemostatic and rehydration therapy. Analysis of stone components suggested anhydrous uric acid. Afterward, he returned to the plateau to resume his previous work and no recurrences were found upon regular re-examinations.

\section{Discussion And Conclusions}

In this report, we present, for the first time, two cases of patients with HAPC who were born at low altitudes and developed ureteral calculi while residing at high altitudes. According to the chronic mountain sickness (CMS) Qinghai integral standard of the 6th Plateau Medicine Conference in 2004 (6), the CMS integral of patients 1 and 2 were 8 and 12 points, respectively. Both cases presented with acute ureteral colic as the first symptom and increased serum uric acid levels. In light of the aforementioned data, we speculate that polycythemia may be a risk factor for ureteral calculi in individuals who migrate to the highlands. To the best of our knowledge, this is the first report that presents the occurrence of HAPC with ureteral calculi in migrants to the highlands.

The etiology of ureteral calculi remains unknown and is related to geographical locations, climatic environments, dietary habits, occupations, and basic diseases (7). Ureteral calculi account for $65 \%$ of urinary calculi and the incidence has been rising recently (8). It is well accepted that hyperuricemia significantly increases the incidence of urinary calculi (9). When the uric acid concentration is supersaturated, it gets deposited in the renal tissues in the form of sodium urate crystals. Researchers have found that there was a $75 \%$ incidence rate of urinary tract stones in patients with hyperuricemia caused by rare single-gene enzyme disorders (10).

Uric acid is the end-product of purine nucleotide metabolism and approximately two-thirds of uric acid excretion occurs through the urine (11). Hyperuricemia is a common condition in patients with HAPC and multiple factors contribute to this process. Excessive erythrocyte proliferation and apoptosis of HAPC may contribute to increased uric acid levels (12). Hyperuricemia is also commonly found in patients with erythrocytosis secondary to lung diseases and polycythemia vera $(13,14)$, which indirectly indicates the correlation between erythrocytosis and uric acid. Moreover, the filtration function of the kidneys can be inhibited by increased blood viscosity. In addition, high-altitude hypoxia increases the concentration of lactic acid, especially in patients with HAPC, which can competitively inhibit the excretion of uric acid (15). 
A high uric acid concentration and the persistent low $\mathrm{pH}$ of urine play a key role in the pathogenesis of uric acid calculi (5). Urine $\mathrm{pH}$ can affect the formation of different types of urinary stones. Under normal circumstances, urine $\mathrm{pH}$ is approximately 5.7-6.3. Consequently, a uric acid calculus is easy to form when the urine $\mathrm{pH}$ is $<5.5$, while calcium phosphate and struvite calculi readily form when the urine $\mathrm{pH}$ is high $(16,17)$. In addition to pure uric acid stones, uric acid or sodium urate precipitation provides a nidus for the subsequent formation of calcium oxalate stones, thereby further contributing to the overall incidence of stones (18). Individuals are at risk of developing hyperventilation and respiratory alkalosis when they are exposed to acute hypoxia, although their $\mathrm{pH}$ is kept within a normal range (19). Moreover, the formation of urinary calculi has a relatively slow progress rate, and hence, acute hypoxic exposure has a limited effect on urinary stone formation. However, for patients with HAPC who are exposed to chronic hypoxia, a high uric acid concentration plays an important role in the formation of uric acid stones. Moreover, the concentrations of uric acid metabolites in such patients are increased, and uric acid crystals are apt to precipitate and deposit in the kidneys. A urinary calculus is formed in patients with HAPC, which in turn aggravates the renal excretion function that can ultimately lead to uric acid formation thereby continuing a vicious circle.

The prevention of ureteral calculi associated with HAPC is particularly important for several reasons. First, in routine blood investigations, the serum uric acid level and 24-h urinary $\mathrm{pH}$ should be regularly monitored in individuals who have migrated to the high-altitude areas. Second, drinking plenty of water is recommended because a large amount of liquid intake has a protective effect on the incidence of urolithiasis. Third, the dietary structure should be appropriately adjusted by reducing the intake of foods with high purine content. It is worth noting that type II diabetes, metabolic syndrome, and obesity are associated with hyperuricemia, and are also the risk factors for uric acid stone formation (20). Therefore, people with these diseases entering the plateau should monitor the uric acid levels in order to reasonably prevent the occurrence of urolithiasis.

Urolithiasis associated with HAPC might be a public health concern worldwide. Implementation of a system to monitors serum uric acid levels in individuals residing in high-altitude areas could help prevent ureteral calculi associated with HAPC. Meanwhile, publicity and education on this matter should be strengthened to improve the dietary structure and develop the habit of drinking plenty of water. Further research on the underlying mechanisms of urinary stone formation in patients with HAPC, including stone analysis and 24-h urine composition analysis could provide useful information regarding the association between urinary stone formation and HAPC.

\section{Abbreviations}

HAPC: High-altitude polycythemia; JAK2:Janus kinase 2; KUB: abdominal horizontal position radiograph; IVP: intravenous urography; CMS: chronic mountain sickness

\section{Declarations}




\section{Acknowledgements}

Not applicable.

\section{Funding}

This study was supported by the Funding for Returned High-level Overseas Talents of Ministry of Human Resources and Social Security of China (no. 2017-200囚no. 2017-805), and Basic Research for Application of science and technology department of Qinghai Province (no. 2019-ZJ-7081).

\section{Availability of data and materials}

All the data supporting the conclusions of this article are included in the present article.

\section{Authors' contributions}

SC, LJ, and MY designed the study. KM performed the literature search. SC and MY performed the medical records analysis and interpretation. MY and LJ drafted the manuscript and revised the main body of the manuscript. SC, LJ, RG, and TW revised the final manuscript, instructed the writing of the manuscript. All authors read and approved the final manuscript.

\section{Ethics approval and consent to participate}

Ethical approval is not required because this is a single case report that involves only a retrospective review of medical records and does not include data that can identify the patient. Written informed consent was obtained from the patient for participation in this case report.

\section{Consent for publication}

Written informed consent was obtained from the patient for publication of this case report and any accompanying images. A copy of the consent document is available for review by the editor of this journal.

\section{Competing interests}

The authors declare that they have no conflicts of interest.

\section{References}


1. Wang ZK, Liu FJ, Ye SL, Jiang P, Yu XC, Xu J, Du X, Ma L, Cao HJ, Yuan C, Shen YZ, Lin FZ, Zhang R, Li CQ. Plasma proteome profiling of high-altitude polycythemia using TMT-based quantitative proteomics approach. J Proteomics. 2019;194:60-9.

2. Li M, Ci RCM. Changes of serum uric acid in patients with polycythemia at $4300 \mathrm{~m}$ altitude. Tibetan Medicine. 2010;2:43-4.

3. Wang SY, Lou XM, Li MY, Zhang XF, Zhou QQ. Analysis of the risk factors of uricemia in men at high altitude. Journal of High Altitude Medicine. 2014;04:32-4.

4. Yu HT, Ci RCM, Jian XJ, Bian P, Yang XX, Da WCR. Effect of hemoglobin concentration on serum uric acid level in physical examination population at high altitude. Tibet Science and Technology. 2018;12:47-48+51.

5. Liu CJ, Wu JS, Huang HS. Decreased associated risk of gout in diabetes patients with uric acid urolithiasis. J Clin Med. 2019;8:1536.

6. Leon-Velarde F, Maggiorini M, Reeves JT, Aldashev A, Asmus I, Bernardi L, et al. Consensus statement on chronic and subacute high altitude diseases. High Alt Med Biol. 2005;6:147-57.

7. Trinchieri A, Montanari E. Prevalence of renal uric acid stones in the adult. Urolithiasis. 2017;45:55362.

8. Corbo J, Wang J. Kidney and ureteral stones. Emerg Med Clin North Am. 2019;37: 637-48.

9. Kong WY. A survey of the relationship between urolithiasis and hyperuricemia in lianshan area. Practical Clinical Journal of Integrated Traditional Chinese and Western Medicine. 2017;09:21-3.

10. Moe OW, Abate N, Sakhaee K. Pathophysiology of uric acid nephrolithiasis. Endocrinol Metab Clin North Am. 2002;31:895-914.

11. Pehlivanlar-Kucuk M, Kucuk AO, Ozturk CE, Er MC, Ulger F. The association between serum uric acid level and prognosis in critically ill patients, uric acid as a prognosis predictor. Clin Lab. 2018;64:1491500.

12. Jefferson A J, Escudero E, Hurtado ME, Kelly JP, Swenson ER, Wener MH, et al. Hyperuricemia, hypertension, and proteinuria associated with high-altitude polycythemia. Am J Kidney Dis. 2002;39:1135-42.

13. Chen M, Shen Q, Gu J, Yu GY. Hyperuricemia secondary to polycythemia vera. Hematology branch of Chinese Medical Association. Papers of the Eighth National hematology academic conference of Chinese Medical Association: 244. 2004.

14. Sunnetcioglu A, Gunbatar $H$, Yildiz $H$. Red cell distribution width and uric acid in patients with obstructive sleep apnea. Clin Respir J. 2018;12:1046-52.

15. Ba YG, Zhang RX, Qin F, Xin HY, Xie XY, Sun H. Study on the relationship between high altitude polycythemia and renal damage. J High Alt Med. 2017;01:15-8.

16. Das P, Gupta G, Velu V, Awasthi R, Dua K, Malipeddi H. Formation of struvite urinary stones and approaches towards the inhibition-A review. Biomed Pharmacother. 2017;96:361-70. 
17. Ennis $\mathrm{JL}$, Asplin JR. The role of the 24-h urine collection in the management of nephrolithiasis. Int $\mathrm{J}$ Surg. 2016;36:633-7.

18. Maalouf NM, Cameron MA, Moe OW, Sakhaee K. Novel insights into the pathogenesis of uric acid nephrolithiasis. Curr Opin Nephrol Hypertens. 2004;13:181-9.

19. Sato M, Severinghaus JW, Powell FL, Xu FD, Spellman MJ Jr. Augmented hypoxic ventilatory response in men at altitude. J Appl Physiol. 1992;73:101-7.

20. Khan SR, Pearle MS, Robertson WG, Gambaro G, Canales BK, Doizi S, et al. Kidney stones. Nat Rev Dis Primers. 2016;2:16008.

\section{Figures}
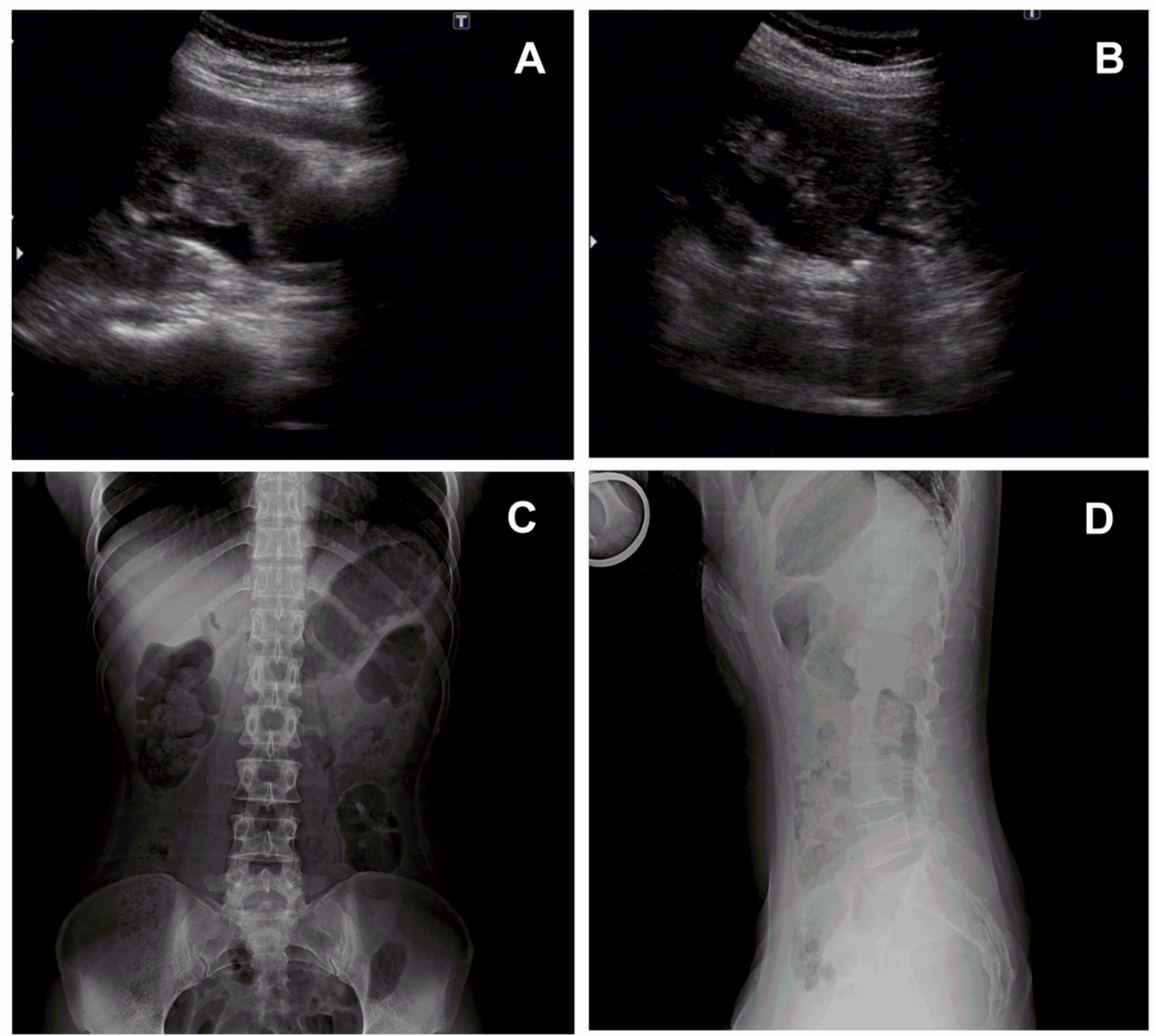


\section{Figure 1}

The Urological Doppler ultrasound and radiograph of KUB of patient 1. (A and B) The Urological Doppler ultrasound revealed hydronephrosis of the right kidney and right proximal ureteral calculus. (C and D) The abdominal horizontal position radiograph in the right upper segment of the ureter deformable area showed high-density shadow because of the calculi.
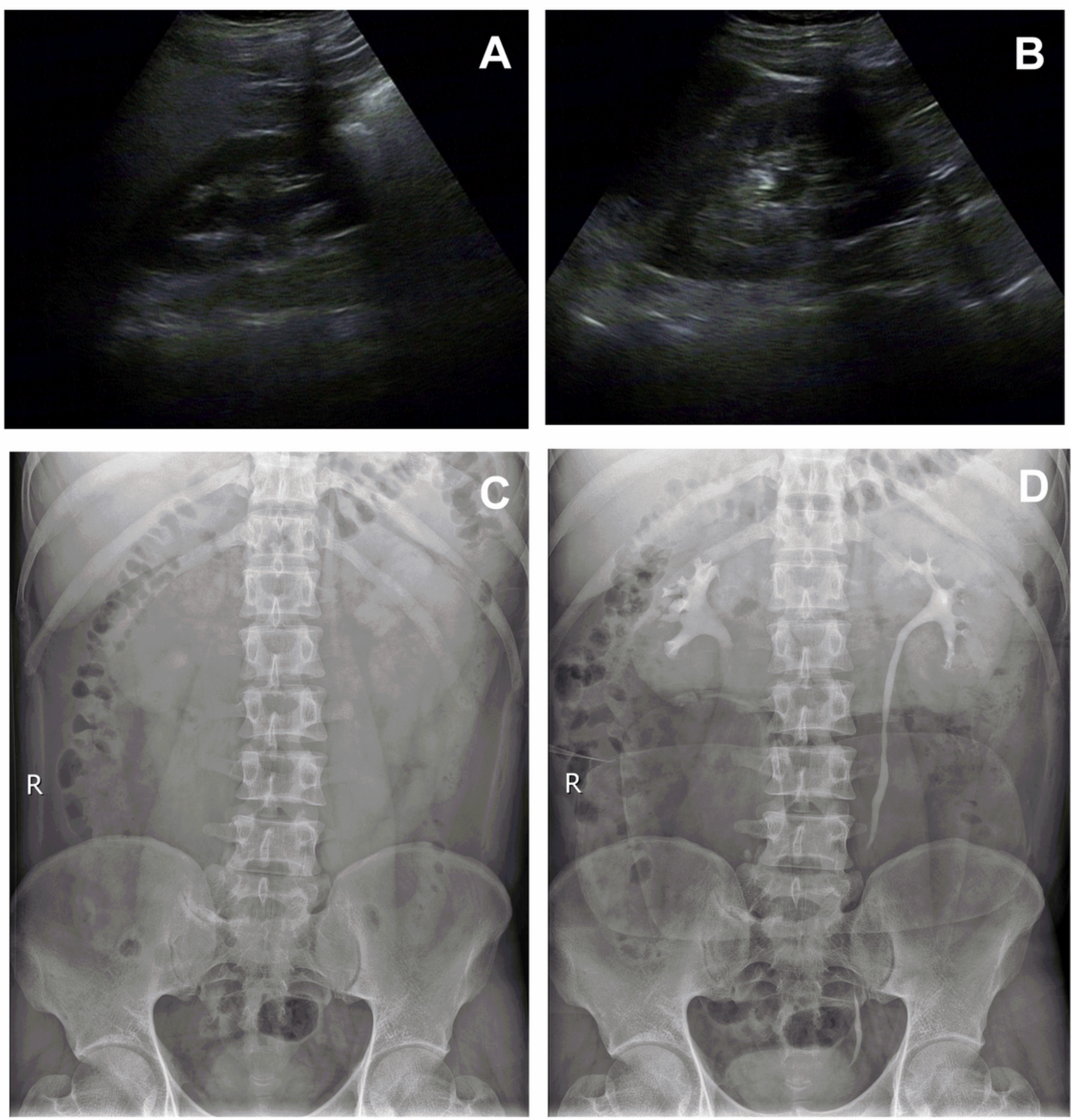

Figure 2 
The ultrasonography of the urinary system, KUB, and IVP of patient 2. (A) Right hydronephrosis. (B) Right ureterectasia. (C) KUB showed no positive urinary calculi in the bilateral renal area and ureteral path. (D) IVP showed right hydronephrosis and dilatation in the upper and middle segment of the right ureter. 